\title{
Restitution des restes humains. Table ronde au festival de cinéma Rochefort Pacifique
}

\section{(2) OpenEdition \\ Journals}

Édition électronique

URL : http://journals.openedition.org/jso/6883

DOI : $10.4000 /$ jso.6883

ISSN : $1760-7256$

Éditeur

Société des océanistes

Édition imprimée

Date de publication : 15 octobre 2013

Pagination : 89-102

ISBN : 978-2-85430-035-2

ISSN : 0300-953x

Référence électronique

"Restitution des restes humains. Table ronde au festival de cinéma Rochefort Pacifique », Journal de la Société des Océanistes [En ligne], 136-137 | 2013, mis en ligne le 23 octobre 2013, consulté le 15 avril 2020. URL : http://journals.openedition.org/jso/6883 ; DOI : https://doi.org/10.4000/jso.6883 


\section{Restitution des restes humains. Table ronde au festival de cinéma Rochefort Pacifique}

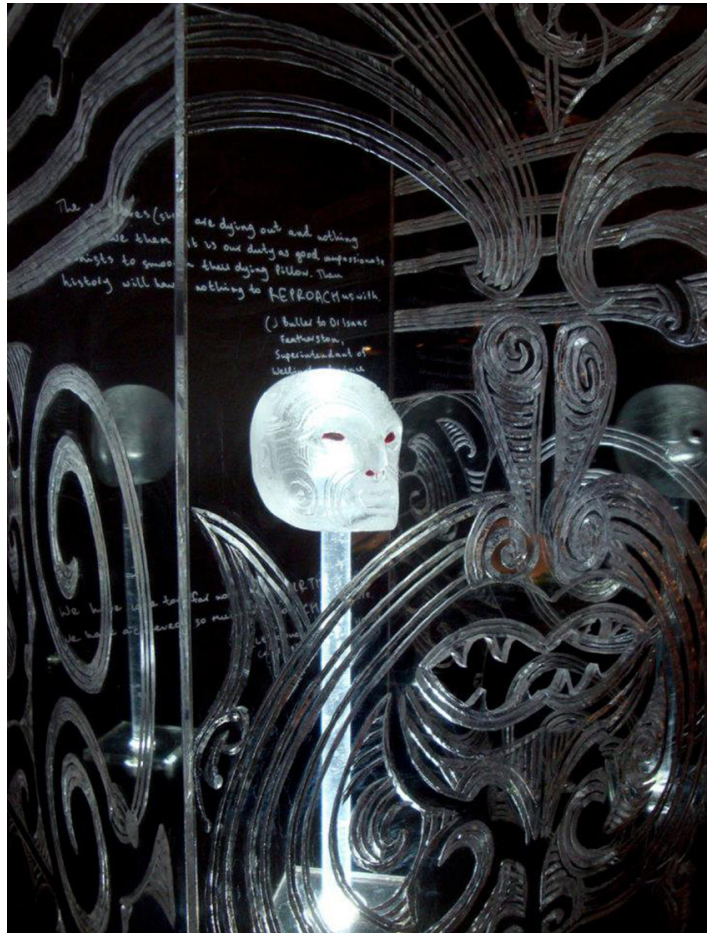

Рното 1. - Une vitrine sculptée par Georges Nuku avec une tête māori en plexiglass, exposée à la galerie Meyer à Paris en 2011 (@) Cosaert@the image paradox)

Dans le cadre du $7^{e}$ Festival de cinéma Rochefort Pacifique, qui a mis le cap cette année notamment sur la fragilité de la vie en PapouasieNouvelle-Guinée face au mutations du monde, s'est tenue le 10 mai 2013 au Palais des Congrès de la ville une table ronde sur la Restitution des restes humains. Faisant suite à la projection $\mathrm{du}$ documentaire La fabuleuse histoire de la tête māori (2011, 52', dir. Philippe Tourancheau), les débats ${ }^{1}$ de cette table-ronde animée par Gilles Luneau, rédacteur de Global Magazine, ont rassemblé Georges Nuku, artiste māori, Emmanuel Kasarhérou, ancien directeur de l'agence pour le développement de la culture kanak (ADCK), chargé de mission pour l'outremer au musée du quai Branly, Sébastien Minchin, directeur du muséum de Rouen, Claude Stéfani, conservateur du musée Hèbre de Saint-Clément (Rochefort), Denis Roland, attaché de conservation du patrimoine au musée national de la Marine et à l'école de médecine navale de Rochefort.

Gilles Luneau. Quelques mots d'abord à propos du film que nous venons de voir : quel film et quelle aventure! La première chose que je voudrais souligner est que ce film nous envoie directement au moment de l'aventure coloniale européenne que l'on prend en pleine face. La deuxième chose qui est celle qui me marque le plus, ce qu'on découvre, c'est le statut de choses que les conservateurs accordent aux restes humains dans la culture patrimoniale. D'où la première question que je vais vous poser : est-ce que cette affaire de la restitution des têtes māori a permis de bouger quelque chose dans le rapport au patrimoine dans les musées?

Sébastien Minchin. Oui et non en fait. Cela a permis de faire avancer quand même quelque peu la problématique, dans le sens où, depuis un certain nombre d'années maintenant, les musées en France doivent faire l'inventaire et le récolement de leurs collections de façon à savoir mieux ce que l'on a dans nos collections. À cette occasion, bon nombre de musées et de collègues, et nous même au sein du muséum de Rouen, ont pu faire le point sur l'ensemble des restes humains que l'on avait. En fait, on a redécou-

1. Les débats ont été enregistrés, puis retranscrits par Isabelle Malaval de AMK France. Isabelle Leblic et Jessica De Largy Healy (pour le JSO) en ont fait l'adaptation pour la publication qui a été soumise aux intervenants pour d'éventuels compléments ou corrections. Que tous soient ici remerciés. Toutes les notes sont de la rédaction. Il faut remercier Estelle Castro qui a bien voulu se charger de faire la traduction simultanée des interventions anglaises en français. 
vert "plein de cadavres dans les placards", si je puis dire. Parce qu'à l'époque, au XVIII ${ }^{\mathrm{e}}$, au XIX siècles, les restes humains, les cadavres faisaient complètement partie des collections de la vie, de la mort, enfin voilà, cela faisait partie des choses qui étaient présentées au public, aux visiteurs.

Et puis, au cours du $\mathrm{xx}^{\mathrm{e}}$ siècle, je pense qu'avec les deux conflits mondiaux, les choses se sont un peu compliquées et on a mis, tout au moins dans beaucoup de musées (pas forcément dans le musée de la médecine mais dans beaucoup de musées), les restes humains dans les placards, au fin fond des réserves, le plus loin possible, pour ne plus les présenter au public. Nous, à Rouen, c'était comme ça. Et du coup, on a perdu la trace de ces restes humains. Maintenant, le fait d'avoir fait cet inventaire, ce récolement, a permis que l'on regarde un petit peu plus dans les réserves et que l'on redécouvre le contenu de ces placards. Cela nous a permis, justement, de faire un point sur ces restes humains et de voir tout ce que l'on aborde quand on parle de restes humains. Comme vous l'avez vu dans le film, ça va des crânes, des os à des morceaux de peau... à des choses de ce style-là.

L'idée de cette démarche, dès le départ, tout comme celle de ce débat, n'était pas forcément d'être pour ou contre toutes les restitutions, ce n'était pas du tout ça! C'était vraiment de dire, voilà, on a ces éléments-là dans nos collections, est-ce que l'on peut considérer que ce sont des biens muséographiques ou pas? Et est-ce qu'ils ont encore le statut de restes humains ou pas? Est-ce qu'on peut les présenter de la même façon que n'importe quel objet? Est-ce qu'on les conserve de la même façon, que ce soit dans un musée en province ou dans les musées nationaux ayant plus de moyens au niveau de la conservation, comme par exemple au musée du quai Branly, au musée de l'Homme? Quand on est comme moi à la tête d'un petit musée en province, on se retrouve face à des restes humains, comme par exemple les foetus, qui ont été abandonnés pendant des décennies sans formol, sans alcool... et qui ont donc séché, pourri, moisi, ainsi de suite..., ça amène plein de questions par rapport à tout ça! Qu'est-ce qu'on en fait ? Comment les conserve-t-on? Est-ce que, en tant que directeur d'une structure de province, je suis en capacité de conserver dans de bonnes conditions, à la fois patrimoniales si je considère que c'est un « objet » et éthiques de dignité humaine et de respect si je considère que c'est un reste humain? Et puis, on arrive à la dernière question, celle posée par le film, celle de toute cette démarche : jusqu'où on peut aller? Qu'est-ce que je peux faire de ces restes? Est-ce qu'ils vont rester $a d v i$ - tam aeternam dans une réserve ? Est-ce que je vais les exposer de temps en temps ? Et est-ce que je ne peux pas aller plus loin ou pas, vers une démarche de restitution quand on me le demande, quand un peuple autochtone le demande? Estce qu'on prend en compte sa demande? Voilà, c'était un peu toutes ces questions-là !

Gilles Luneau. Je me tourne vers Denis Roland. Vous avez des restes humains au musée de la Marine... quelle est votre position sur ces questions?

Denis Roland. Oui, je pense que les lignes ont quand même un peu bougé, pas forcément sur les notions de conservation proprement dites, mais sur ce que, dans les musées, il est convenu d'appeler la médiation, qui est un terme bien pratique, parce qu'il recouvre des tas de choses. Comme il ne veut pas dire grand-chose, on y met un peu ce que l'on veut! Puis c'est plutôt la façon dont on parle de ces questions qui a changé. Dans le film, il y a un mot qui revient souvent, qui est celui de respect, qui est effectivement, je pense, une notion centrale dans ce débat sur les restes humains, que ce soit par rapport à des biens d'autres peuples, ou que ce soit par rapport à des collections d'anatomie humaine, comme c'est le cas à l'école de médecine navale. C'est une évolution du droit qui a été portée par cette affaire des têtes māori, mais aussi par d'autres affaires qui n'ont rien à voir. Vous avez tous dû entendre parler de Gunther Von Hagens, qui est cet anatomiste qui a fait des expositions extrêmement spectaculaires avec des cadavres et qui ont été montrées et continuent d'ailleurs de l'être avec grand succès [presque] partout dans le monde! Mais qui ont été interdites en France à Paris en 2010, d'ailleurs précisément au même moment où se déroulaient les épisodes essentiels de l'affaire des têtes māori ${ }^{2}$. Donc toutes ces choses se mélangent beaucoup et ont des influences sur le droit, qui lui-même s'adosse sur la loi sur la bioéthique. Bref, tout cela a créé une situation assez compliquée.

Donc la vraie réponse, je pense, en tout cas en ce qui nous concerne, ce n'est certainement pas de cacher ce que l'on a, mais plutôt au contraire de le montrer, car je pense que plus on cache, plus on génère des problèmes. Si on cache, on laisse le champ libre à l'imagination et aux fantasmes les plus débridés, ce qui, dans certains cas, est très bien, mais pour ce qui nous concerne ici, n'est pas forcément idéal. En revanche, on accompagne les visiteurs en leur expliquant la collection car, en gros, notre métier de conservateur, c'est de comprendre ces collections, quelles que soient leur histoire et leur nature, et de dire

2. Voir le dossier « Restitution des têtes tatouées momifiées māori » publié dans le JSO 134, avec les articles de Natacha Gagné, Roger Boulay, Philippe Peltier et Magali Mélandri ; et, dans ce volume l’article de Natacha Gagné « Musées et restes humains : analyse comparée des cérémonies māori de rapatriement en sols québécois et français ». 
ce qu'on en a compris aux visiteurs. Alors, c'est effectivement des médiations qui sont parfois assez contraignantes, assez lourdes et qui consistent, en fait, à imposer des visites guidées. Mais cela permet de tout montrer, y compris les foetus dans les bocaux qui sont devenus des espèces d'emblèmes des musées des horreurs, alors qu'en fait, ce sont des pages fondamentales de l'histoire de notre civilisation, parce que c'est là que se sont construites certaines visions du monde dont nous sommes directement les héritiers.

Claude Stéfani. On présente aussi des restes humains au musée Hèbre de St-Clément, notamment dans notre collection du Pacifique. Le " matériau humain » est en effet très utilisé par les cultures de cette région, et pas uniquement en Nouvelle-Zélande, mais aussi par exemple aux Marquises où l'on se servait des cheveux et des os humains. Au musée Hèbre de St-Clément, on présente des objets marquisiens de ce type. Le cas n'est pas unique; si vous allez au musée de La Rochelle, vous trouverez aussi ce genre d'objets. À Rochefort, on expose toujours un objet māori fait en " matériaux humains ", un pendentif constitué d'une vertèbre cervicale gravée.

Mais le problème spécifique des têtes māori a été évoqué dès le début et il faut peut-être y revenir, recentrer notre propos, car c'est un cas d'école. Le problème posé était celui de l'ostension ou pas, soit de montrer ou pas la pièce. Il y a d'autres populations autochtones qui ne se sont jamais préoccupées de ce qu'on faisait des restes humains. Pour le Pacifique, l'importance des crânes, de la tête, dans les cultures polynésiennes notamment, mais aussi mélanésiennes, a été évoquée par Roger Boulay. Les crânes subissent souvent des préparations très particulières dans beaucoup de ces cultures. Ainsi, on a vu chez les Māori une forme de momification des têtes ; aux îles Marquises, les crânes sont souvent enveloppés dans du tapa, ou alors pourvus de dents de cochons ; aux îles Salomon, ils sont entourés d'un réseau de coquillages. Les traitements sont multiples. Et les seuls jusqu'à présent à s'être préoccupés de ces objets-là, ce sont les Māori qui, très tôt, ont demandé à ce qu'ils ne soient plus présentés. Cela n'a pas été respecté partout. Je me rappelle, au muséum de Lyon en 2000, une tête māori présentée sans que le conservateur de l'époque ne soit vraiment informé de la demande du gouvernement de Nouvelle-Zélande. Elle a été retiré peu après notre passage. Se posait la question de la mettre en réserve ou d'en faire autre chose. Notre grande crainte, en tant que rsponsables de collections, quand on a connais-

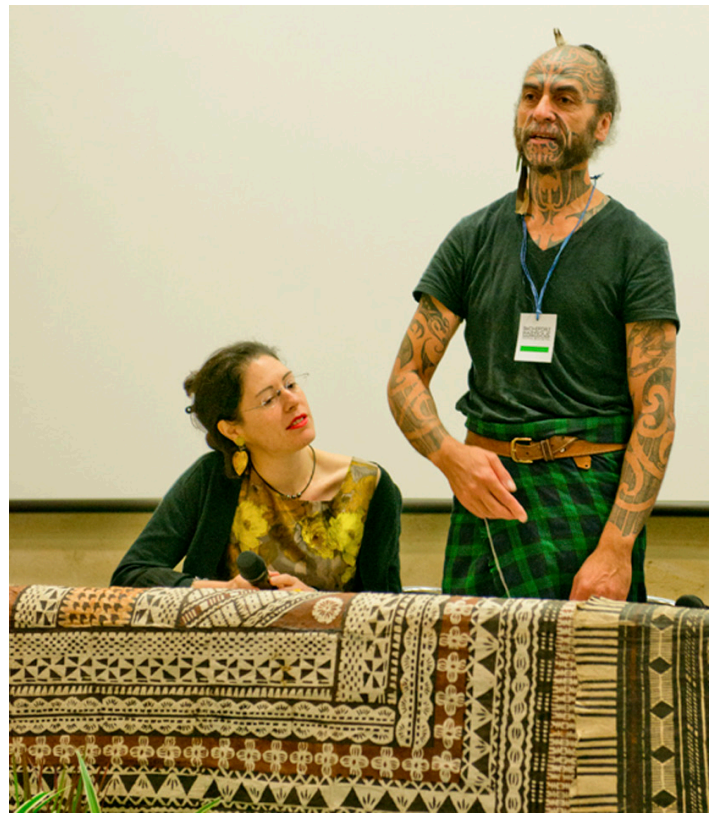

Рното 2. - Georges Nuku et Estelle Castro (cliché de Béatrice Téraube)

sance de l'affaire de Rouen, était que cela fasse " tache d'huile» et que les demandes de restitution se multiplient... Demande totalement fondée de la part des Māori, qui pourraient faire apparaître simultanément des demandes de la part d'autres populations. Il y a eu au musée du quai Branly un grand colloque sur les restes humains ${ }^{3}$ où le responsable du musée du Vanuatu, donc des anciennes Nouvelles-Hébrides, a déclaré que son gouvernement ferait sans doute une demande similaire à celle des Māori pour la restitution des têtes surmodelées et des mannequins funéraires qui sont conservés dans les collections françaises. Et puis, plus tard, la personne s'est en fait rétractée. Ce qui a été assez étonnant comme posture, dans la même journée...

Gilles Luneau. Est-ce qu'il ne faut pas introduire une différence dans le temps, dans la nature, de la manière dont se sont constituées les collections? Je m'explique. Ne faut-il pas différencier ce qui provient, on va dire, des razzias coloniales, qui sont datées - c'est le cas avec l'affaire des têtes māori - et puis ce qui ressort du tas d'os trouvés dans une grotte du paléolithique? Vous voyez ce que je veux dire! Il faut peut-être introduire une différence! Entre ce qui est directement de l'histoire coloniale, et ce qui est des restes humains qui n'appartiennent qu'à eux-mêmes finalement! Qui va réclamer un os de Neandertal ?

3. «Des collections anatomiques aux objets de culte: Conservation et exposition des restes humains dans les musées », 22-23 février 2009, Voir les actes du colloque http://www.quaibranly.fr/fr/programmation/manifestations-scientifiques/ manifestations-passees/colloques-et-symposium/saison-2009/des-collections-anatomiques-aux-objets-de-culte-conservation-et-exposition-des-restes-humains-dans-les-musees.html. 


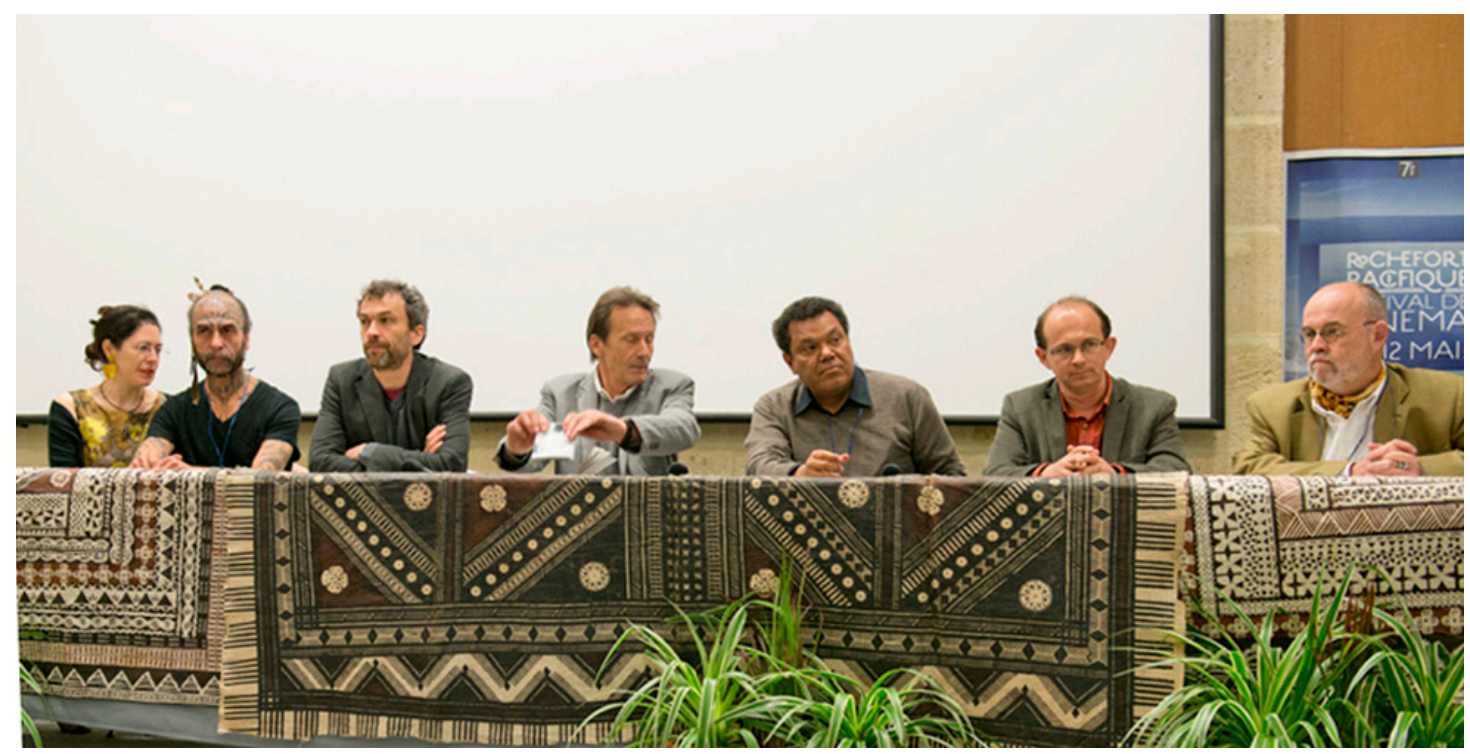

Рното 3. - Vue des intervenants (cliché de Béatrice Téraube)

Emmanuel Kasarhérou. Il me semble que ce qui est intéressant, c'est effectivement de ne pas globaliser la question, parce que la question des têtes māori, comme cela a été souligné, c'est vraiment une demande des Māori soutenue par le gouvernement néo-zélandais. Alors, après, ce qui est intéressant, c'est de s'interroger sur ce qu'il se passe réellement en Nouvelle-Zélande autour de ces demandes, et qu'est-ce que l'on fait ensuite des têtes restituées, soit quel est le fondement finalement de tout cela. Ce que j'observe, c'est qu'actuellement, au musée du quai Branly, on a effectivement en présentation permanente des têtes qui sont papou, ni-vanuatu, des têtes surmodelées. On a des crânes reliquaires des Salomons qui sont en exposition. Si vous y allez, vous les verrez. Donc, il n'y a pas l'idée de retirer tous les restes humains! D'ailleurs, ça a été évoqué. Qu'est-ce qu'un reste humain ? On a parlé des ossements, on a parlé des peaux. Mais est-ce que les cheveux, tous les phanères, tout ce qui est produit par le corps humain quand il est en vie..., est-ce qu'on doit l'étendre à tout ça ? Je pense que le mérite de l'affaire des têtes māori, c'est d'avoir ouvert le débat et d'amener à se poser la question de ce que l'on conservait dans les collections. Et c'est vrai que, souvent, quand on est nommé à la tête d'un musée, on saisit d'abord ce qu'est la collection, la face émergée de l'iceberg, et puis quand on entre dans les réserves, on fait des découvertes.

J'ai commencé ma carrière au musée de Nouméa, j'ai été confronté aussi à des restes kanak en fait qui sont dans les réserves. Il y en avait quelques-uns qui étaient en présentation. Ma première réaction a été d'enlever des vitrines les crânes qui évoquaient les anciens lieux de sépulture kanak pour les remettre en réserve. Mais on a encore des ossements dans ce musée. On a été saisi par ailleurs, au début des années 1990, par des musées américains qui avaient obligation, de par la loi passée sur les restes humains ${ }^{4}$, de s'adresser aux peuples autochtones et de leur demander ce qu'on devait faire des restes humains. J'avais une liste avec une centaine d'omoplates, de tarses, de crânes, toute une série d'os, dont on ne connaissait pas l'origine. Mais simplement que cela venait de Nouvelle-Calédonie. À l'époque, j'étais directeur du musée, la première chose que j'ai faite, c'est de saisir le conseil coutumier, puisqu'on a la chance d'avoir un conseil coutumier en Nouvelle-Calédonie, qui représente les autorités coutumières, à la fois l'aspect moral, mais aussi la légitimité de parler au nom d'un groupe. Ce n'est pas un individu qui peut parler, c'est une assemblée évidemment. Et leur réaction a été de savoir qui étaient ces gens. Quand je leur ai dit qu'on ne savait pas, que c'était simplement des Kanak, et que, d'ailleurs, au XIX ${ }^{\mathrm{e}}$ tout les autochtones du Pacifique étaient nommés Kanak - ce n'est qu'aujourd'hui que le mot Kanak est resté pour les peuples autochtones de Nouvelle-Calédonie - la réaction a été immédiate. On ne va rien en faire! C'est-à-dire que quand les choses sont tombées dans l'anonymat, on ne peut pas gérer cette question d'un point de vue coutumier. On pourrait le faire si on avait une connaissance de l'individu. À ce momentlà, on peut remonter à son clan. De son clan, on peut retrouver ses maternels, et vers ces maternels, on peut commencer à envisager de faire une sorte de coutume post-mortem de deuil. Ce 
qui est la manière coutumière de gérer les corps humains dans cette culture aujourd'hui. La réponse qu'on a faite aux musées américains qui nous avaient saisis, c'était : gardez-les! Vous avez des restes humains kanak, très bien. Ils sont anonymes, vous les avez pris, gardez-les, on ne sait qu'en faire aujourd'hui ! Vous nous saisissez avec une question qui nous surprend et qui n'entre pas dans notre manière de régler ces questions. C'est une réponse qui a été faite, il y a une vingtaine d'années maintenant. Si on était saisi à nouveau par un musée pressé par une loi nationale de restituer des corps, quelle serait la réaction aujourd'hui ? Ce que j'observe, c'est qu'il y a une sorte de globalisation aussi des réponses autochtones. Jusque-là, on avait des peuples différents les uns à côté des autres, chacun avec sa relation au corps, sa relation à la mémoire, sa relation aux restes humains qui est spécifique.

Aujourd'hui, du fait de ces lois américaines, ensuite de ces prises de position māori, on observe une forme de globalisation finalement de la réponse en relation à la question des restes humains. Et donc, ça fait débat sur des forums internationaux, alors que, jusqu'à présent, c'était plutôt des choses qui se géraient au cas par cas. Pour ma part, je pense que c'est important de dire les choses. Que lorsque l'on a effectivement trouvé une tête māori dans son musée, c'est important de le faire savoir, c'est important d'en connaître l'histoire et d'entrer en relation avec le peuple en question pour savoir s'il y a une demande ou pas.

En ce qui concerne le musée du quai Branly, pour l'instant, on a effectivement en présentation des objets, qui sont des objets culturels faits avec des restes humains. Il y a d'ailleurs une exposition en ce moment - je crois que c'est un des rares musées en France qui le fait encore qui s'appelle Cheveux chéris, dans laquelle on peut voir des têtes shuars, c'est-à-dire des têtes réduites jivaros, qui sont des objets qui ont aussi été retirés des présentations depuis longtemps. Et cela, dans un but de connaissance, de partage ! Maintenant, si un jour ces populations en viennent à réclamer qu'on les retire d'expositions et qu'on les restitue... ce sont des choses qu'il faudra voir au fur et à mesure des demandes. Ce que je pense, c'est qu'il n'y a pas de réponse globale en fait et qu'il faut regarder les choses chaque fois au cas par cas, comme ça a été fait jusque-là.

Gilles Luneau. Je me tourne vers Georges Nuku. Déjà merci de nous faire l'honneur de votre présence. On a bien compris l'importance que cela représentait pour vous de récupérer les têtes, ce qui, pour nous, est une vraie découverte. Vous nous avez mis face à notre propre histoire. J'ai envie de vous demander ce que vous a appris au cours de cette aventure, sur vous comme sur nous...
Georges Nuku. Merci beaucoup de l'opportunité que j'ai ici de vous saluer, les gens de Rochefort. Ce n'est pas poli de parler d'abord à un public sans d'abord dire bonjour. Je voudrais aussi saluer mes frères du Pacifique. Mes pères, mes mères, mes cousins, je vous salue. Je suis dans votre empire. Mon empire appartient à Élisabeth. Et je suis dans votre empire. Je passe des vacances de colonisateur, parce que j'ai décidé de faire de la France mon pays. Donc, je vous salue. Je voudrais aussi saluer tous vos défunts et je voudrais que vos défunts rencontrent, rejoignent les miens et qu'ils soient présents et qu'ils retournent à Hawaiki, dans leur patrie.

Cela nous laisse ici, nous, les vivants. Je nous salue aussi, nous, les vivants. Je voudrais aussi saluer les personnes qui parlent à cette table ronde et tous les organisateurs du festival. Merci beaucoup de m'avoir invité à ce festival.

Maintenant, cette question de la restitution des restes humains... Il y a beaucoup de questions qui ont été abordées, très importantes et très intéressantes. J'ai l'avantage de parler après leurs interventions. Comme ça a été dit, nous devons être très attentifs et faire très attention à ne pas mélanger la question des mokomokai avec la question des restes humains. Tout d'abord, je voudrais dire qu'ils n'ont pas été collectés. Collectés, cela voudrait dire que les gens seraient venus pour les prendre, et ça, ce n'est pas vrai. Au départ, les échanges et le commerce ont été faits par les Māori. C’est nous qui avons établi le prix pour ces échanges et c'est nous qui avons déterminé comment ces échanges se passeraient. Ça, c'est très important. Parce que ça parle de l'agentivité, du fait que nous avons été acteurs en tant que peuple. Et cela en dit beaucoup sur notre capacité d'être des acteurs du changement. Quand vous êtes sur la plage en pagne végétal et que vous voyez un bateau de guerre pointant ses quarante canons vers vous, cette notion de pouvoir est quelque chose qui est très tangible.

Je peux vous dire que mes ancêtres avaient une compréhension très développée de ce qu'était le pouvoir. Même si je comprends que le film (La Fabuleuse histoire de la tête maori) a vraiment de bonnes intentions, pour moi, c'est un film qui est trop bien pensant et qui ne prend pas en considération les paradoxes, l'ironie de toute cette histoire fascinante.

Nous avons vendu nos ancêtres pour des armes! Nos ancêtres étaient d'accord pour faire cela. Nos ancêtres viennent d'une situation, de conditions dans laquelle la guerre c'est la vie. Et la vie, c'est la mort. Pour exprimer les choses d'une autre manière, cela en fait nous relègue à un statut de victimes. Je ne peux pas me faire le défenseur de cette idée. Effectivement, c'est une histoire fascinante, ça fait référence à une histoire turbulente et très intéressante. Cette collision de deux mondes qui se sont rencontrés et qui se 
sont fait face. D'une collision d'une culture qui ne connaissait pas l'existence des métaux et qui s'est confronté au monde de l'âge industriel. Ces gens ont réagi très rapidement. Ils se sont tout de suite embarqués sur les bateaux qui arrivaient, ils sont allés en Australie, en Afrique du Sud, en Angleterre, en Californie et ils ont vu la situation d'autres peuples autochtones, notamment les Aborigènes en Australie. Ils ont été convaincus que les Anglais et les Européens viendraient prendre le pays par les armes. Ils n'étaient pas étrangers à cette notion, ils ont compris tout de suite ce qui allait se passer parce qu'ils étaient des guerriers depuis mille ans.

Ils savaient exactement ce qui était en jeu et ils ont voulu intégrer ce qui était en train d'arriver et non pas être intégrés dans ce qui arrivait. Être des agents d'intégration. Ce pour quoi je vous dis tout ça, c'est pour vous donner le contexte de pourquoi ces objets sont dans vos musées.

Ce sont des choses dont il faut parler à propos de la question que vous avez soulevée. Et pour ce qui est de notre peuple, c'est vrai que nous sommes différents d'autres communautés. Quand c'est une question de vol, effectivement, il faut rendre ce qui a été volé. Et c'est valable pour tout le monde. Mais pour nous, de mon point de vue, demander le rapatriement des restes humains n'est pas pour moi un point de vue que je partage. Je respecte le point de vue et les bonnes intentions des Māori qui veulent ces restes comme je respecte aussi les points de vue des personnes qui ont ouvré pour que cette restitution ait lieu. Mais il y a plusieurs points de vue.

Beaucoup de questions sont abordées dans ce film. Mais le fait que les objets puissent être restitués aux régions d'origine n'est pas possible. Les meilleurs artistes en fait voyagent tout autour du monde, parce qu'on fait appel à leurs services. Et donc on ne tient pas compte de cet entremêlement de facteurs, ça rend les choses trop simples. Et c'est quelque chose qui nous retire notre pouvoir. C'est ça aussi qui est dans le film, avec la question du tatouage post-mortem. Il faut comprendre ce qui s'est passé, ces chefs ont beaucoup de vanité, comme moi d'ailleurs. Et donc, ils voyageaient, ils voulaient que les artistes leur fassent des tatouages à nouveau après leur mort. Ça, c'était leur souhait. Parce que c'est très important d'avoir une belle apparence dans la mort. La question du mokomokai parle de tout cela. Cela parle de l'audace des Māori qui pensent qu'ils peuvent triompher de la mort de cette manière et qu'on devient encore plus beau après la mort. C'est important d'avoir de l'allure, je vous assure. Et, d'une certaine manière, cela me fait mal de voir ces têtes dans des boîtes! Si c'était moi qui devais décider, je voudrais qu'elles soient montrées !
Dans des contextes cérémoniaux, ces têtes étaient montrées sur des javelots avec des costumes magnifiques et des oreilles percées comme moi - et toutes les parures qui vont avec le fait d'être chef, et des coiffes de plumes. Tout cela était fait pour des raisons bien précises, comme le fait qu'elles étaient séchées pour être légères afin que la moindre brise les touchant les fasse bouger. Cela était vu comme une forme de divination. Ainsi, quand il y avait une discussion politique, elles bougeaient un peu. Et les gens cherchaient ces signes qui étaient très importants, qui nous donnaient des indications sur le choix à faire. Vous pouvez appeler ça de la superstition, mais les artistes pensent que tout cela est important. Et ils croient dans ces choses. Pour moi, quand je les ai vues revenir dans leurs boîtes et que les gens pleuraient - et je comprends pourquoi ils pleuraient, ils disaient qu'il fallait qu'ils reviennent à la maison, qu'ils reviennent chez nous -, moi j'ai dit: personne n'a demandé aux ancêtres! Et je vous le dis, les ancêtres m’ont dit très clairement ce qu'il fallait faire. Ils m'ont dit :

"Georges, il faut que tu fasses tout ce qui est en ton pouvoir pour dire pourquoi nous sommes ici, pourquoi nous existons. On ne veut pas être envoyés chez nous et être mis dans une pièce pour être oubliés comme un enfant qui essaie d'oublier un cauchemar. Ça ne sert personne, ni vous, ni nous, personne. »

Mon plus grand regret, c'est que je n'étais pas en France il y a dix ans. Peut-être que les choses auraient été différentes. Je dis cela parce que c'est mon devoir, parce que je vais continuer à me battre pour qu'elles soient montrées de manière publique. Évidemment, la question est : comment est-ce qu'elles seront montrées ? C'est une question qui se pose à tous les conservateurs de musée. Nous avons une responsabilité vis-à-vis du public. Le mokamokai représente l'une des dernières choses que nous avons en notre possession en tant que peuple. C'est une des dernières choses que nous avons pour combattre la globalisation de la médiocrité et de la banalité. C'est une des dernières choses qui nous reste. On ne peut pas faire des publicités avec les têtes māori. Beyoncé ne va pas faire son dernier clip avec une tête māori. Ce ne sont pas des choses qui entrent dans la culture des médias de masse. Et donc, on peut les utiliser de cette manière pour poser des questions sur la banalité qui afflige toute la planète. On est tous menacés par une culture de l'ennui. Et l'opportunité d'essayer d'élever le niveau, pour le public, a été ratée. Parce que c'est plus facile, on simplifie à outrance. Cette pauvreté de réflexion conduit à un appauvrissement de l'esprit. Et donc je suis navré de dire que selon moi, voilà une opportunité qui a été ratée. 
Gilles Luneau. Merci Georges pour cette fenêtre grande ouverte, sur votre culture, qui va permettre d'engager le débat tout de suite, parce qu'on a une salle qui est loin de la médiocrité qui se globalise et loin de la banalité. Je suis sûr que nos amis ont plein de questions maintenant à vous poser, à vous Georges, et à tous nos invités. Il y a des micros qui circulent et il n'y a plus que des mains à lever.

Intervenante. Bonjour, je m'appelle Honorine et je représente l'association des îles Wallis-etFutuna. Permettez-moi d'abord d'adresser des salutations en wallisien. [...] Nous sommes originaires des îles Wallis-et-Futuna et nous répondons à notre frère. Je suis très émue. Nous lui souhaitons la bienvenue et je suis très contente de vous avoir écouté. C'était un message important; nous sommes frères et cousins et on vous remercie beaucoup de venir ici pour y représenter le Pacifique. On vous remercie. Je voulais m'adresser aussi à toute l'équipe, on a passé un très bon moment, c'était un très beau film. Je remercie aussi très émue le réalisateur, c'est un très beau film qui m’a transporté là-bas. [...] Dans le reportage, j'ai senti l'émotion que peut ressentir le peuple là-bas quand il voit arriver le guerrier dans sa maison. Les pleurs des femmes, l'accueil, je le ressentais fort, c'était très fort! Je me suis dit: il revient chez lui ! Il retrouve sa terre natale ! [...] Donc, merci beaucoup pour ce beau film. $[\ldots]$

Intervenante. Ce film ouvre la porte, peut-être, enfin je ne sais pas, à des restitutions [...] par la France de cette époque coloniale où il $\mathrm{y}$ a eu beaucoup de choses de pillées. Et même avant, les guerres dans ces régions. J'ai la chance d'avoir un adhérent dans mon association qui m’a parlé de l'anthropologie du droit. Je ne savais pas du tout ce que c'était. C'est Guillaume Fontanieu. Il a fait un mémoire, il prépare une thèse. C'est vrai que quand on est vivant, on a des droits, quand on est mort, il y a des lois, des règlements. Ce genre de restitution, c'est par le droit. C'est vrai que je suis tentée de parler du retour de la tête d'Ataï en Nouvelle-Calédonie. Mais c'est vrai que ce n'est pas facile. Si le pays est demandeur, estce que la France doit automatiquement rendre les œuvres dans ces pays-là ou c'est suivant les cultures ? Ataï, sa tête a été coupée. Son corps est en Nouvelle-Calédonie et sa tête est ici. Parait-il qu'elle est toujours restée au musée de l'Homme. Mon père a été à l'exposition coloniale en 1931. Il a été enterré ici, j'ai fait l'exhumation et je l'ai ramené dans la tribu où on a fait des obsèques traditionnelles.

C'est vrai que c'est suivant les cultures ! Mais l'accord de Nouméa signé en 1998 prend en compte l'identité culturelle de la Nouvelle-Calédonie et il était marqué «tous les objets culturels de par le monde doivent revenir au pays! " Par exemple, il y a des bâtiments qui n'ont pas de flèches, des choses. Et c'est vrai que ça a été pillé. C'est vrai que dans les cavernes, il y avait les crânes, on a pris les crânes, on les a amenés, les collections particulières où on voit des foetus, de choses dans le monde, mais je crois que ça peut passer par le droit et aussi par le patrimoine mondial! Cette thèse que fait Guillaume Fontanieu, je vous la conseille, parce qu'il a un mémoire sur internet et c'est vraiment très intéressant, cette anthropologie du droit! Merci.

Sébastien Minchin. Je vais vous répondre au niveau du droit sur les restes humains. Vous avez vu qu'il y avait débat. Georges a très bien donné son avis. C'est un vrai débat en interne au sein de la Nouvelle-Zélande et des communautés māori. C'est ça qui était intéressant. C'est-à-dire qu'il n'y a pas une réponse unique. Il y en a plein ! En fonction de là où on se place, en fonction des intérêts des uns et des autres, en fonction de la façon dont on aborde l'histoire. Dès le départ, ce n'était pas d'avoir une réponse sur l'ensemble des restitutions sur tous ces éléments-là. C'était de dire : on a une demande de la part des Māori, de la part d'un certain nombre de communautés māori, et jusqu'où peut-on aller? Est-ce qu'on peut l'ouvrir sur le reste? Bien sûr, on m'a souvent dit que j'avais ouvert la boîte de Pandore. En fait, pas du tout! C'était justement de se servir de cet exemple pour aborder toutes ces questions de façon sereine, dans le dialogue entre les uns et les autres, avec les différents points de vue, ce qui n'avait pas été fait jusqu'à maintenant.

En 2007, quand on a lancé cette démarche, le droit sur les restes humains était nul. Il n'y en avait pas. Parce qu'en fait, dans notre culture occidentale, on a un vrai problème avec la mort. Comme on a un problème avec le début de vie en fait. Très régulièrement, vous avez dans les débats, dans l'actualité, la question qui revient du début de vie : quand est-ce qu'on estime que la vie commence? quand est-ce qu'on estime la notion d'âme? etc. Et puis, vous avez les débats qui reviennent très régulièrement par rapport à la fin de vie, l'euthanasie, la mort, etc. En 2007, quand on a lancé cette démarche, on s'était rendu compte que, dans le droit français, on était sur un vide juridique par rapport aux restes humains. C'est-à-dire qu'il n'y avait aucun texte de loi en 2007 qui aborde le statut des restes humains. Qui est capable de vous dire ce qu'est un reste humain ? Est-ce qu'un reste humain, c'est un cheveu, c'est un ongle, est-ce que c'est un morceau de peau, est-ce que c'est un crâne, un corps entier? On n'a aucune réponse à toutes ces questions-là ! Et c'est ça qu'on voulait montrer, en fait, par cette démarche. Au-delà du fait d'aborder les problématiques de la restitution, 
c'était de montrer et de faire avancer un petit peu la chose par rapport à cette question. Ça l'a fait avancer !'A partir de 2008 - avant même que la loi autorisant la restitution des têtes māori ne soit votée ${ }^{5}$ - dans le code civil, vous avez une protection juridique qui apparaît sur la dépouille. Alors évidemment, elle n’agit pas sur ce qui s'est passé avant, mais elle intervient parce qu'on prend enfin conscience de l'importance d'avoir une protection juridique vis-à-vis de la dépouille. Avant 2007, un cimetière qui a été profané ou un cadavre exhumé n'avaient aucune protection juridique. C'était sa famille, la mémoire de cette personne-là, qui se portait partie civile. À partir de 2008, une protection se met en place. Aujourd'hui, sur les incinérations, vous ne pouvez plus disperser vos cendres n'importe où, parce que c'est réglementé, alors qu'avant, ces cendres pouvaient être jetées dans la mer ou dans les toilettes. Voilà. Cela a permis de faire avancer un petit peu les choses. C'était ça qui était important à notre niveau. C'était de lancer le débat, de lancer le dialogue par rapport à ces questions-là. Il y a des restes humains, qu'est-ce qu'on en fait? Il y a de l'autre côté des demandes de restitution qui sont légitimes, ou pas, et comment considère-t-on qu'une demande de restitution est légitime ou pas! On en avait parlé déjà à l'époque, en 2008, par rapport au musée et à l'université de médecine, là où on avait fait la table ronde à l'époque 6 . On avait abordé la question. On a, dans tous nos musées, la tête du dernier condamné à mort, guillotiné. Monsieur X, violeur, assassin, etc. Et elle est présentée dans la vitrine. Je ne sais pas si c'est encore le cas ici. Cela l'est à La Rochelle. C'est pour vous montrer que les choses ne sont pas aussi simples. C'est-à-dire que là on est même, au niveau de la France, sur le dernier guillotiné à La Rochelle : on a son nom, on vous le présente, ou on le présente pas. On peut le présenter, avec son nom et la raison pour laquelle il a été guillotiné. Mais qu'est-ce qui se passe visà-vis des descendants qui découvrent au cours de leur visite du dimanche la présence de leurs ancêtres guillotinés, violeurs, etc. Ça amène plein de questions. Cela fait cinq ans que l'aventure a commencé et il ne se passe pas une semaine sans qu'on me demande d'en parler. Cette semaine, j'ai encore rempli mon quota! Et il n'y a pas une réponse! Il n'y a pas une réponse ici en France. Je n’ai pas la réponse, Georges n’a pas la réponse. On a chacun des points de vue qui peuvent être différents, mais la différence vis-à-vis du débat qui a eu lieu pendant toutes ces années, c'est que maintenant on se respecte. Ce qui n’a pas été le cas au cours de toutes ces années au niveau des différents ministres et de la façon dont on a été considéré, nous, petit musée. On se respecte et on a travaillé, enfin on travaille ensemble régulièrement avec Georges, sur la façon dont nous pouvons avancer par rapport à cela. On n'est pas forcément d'accord par rapport à la restitution, par rapport au regard qu'on a sur cette loi de restitution des toi moko, sur cette tête māori. Mais comment peut-on travailler ensemble? Et comment peut-on faire avancer les choses, vis-àvis des autres objets du patrimoine que l'on a au sein du musée? On a d'autres objets, vous avez vu la pirogue. L'idée, c'est ça. Comment peuton travailler autour de ces objets-là pour qu'ils soient ambassadeurs de nos deux cultures? Il $\mathrm{y}$ a eu un moment dans notre histoire, où on s'est retrouvé autour de cet objet et qu'est-ce que l'on peut en raconter? L'idée était d'aller plus loin que simplement les quelques informations que l'on avait, qui étaient des informations françaises, rouennaises, de celui qui a ramené cette pirogue. De voir ensemble, comment on peut avancer un petit bout de chemin par rapport à un objet qui nous rassemble tous les deux.

La loi ne nous permet pas de la restituer, mais à un moment ou à un autre, pourquoi est-ce qu'on ne pourrait pas la prêter à un musée sur les îles Chatham par exemple? C'est ça aussi l'intérêt. C'était vraiment d'ouvrir le débat, de l'élever et de l'amener sur différents points de vue, parce que tout ne peut pas se traiter de la même façon.

Emmanuel Kasarhérou. Je me demande si toutes les questions de restitution ne sont pas des questions politiques à la base? Si les musées ne sont pas le lieu de l'expression qui peut se manifester justement en objectivant quelque chose pour pouvoir parler de quelque chose d'autre éventuellement. Et peut-être que les têtes sont un bon prétexte et que les musées sont justement là créés pour être des espaces de négociation, de discussion au travers de ces objets. Je crois que ce qui me paraît dangereux et peut-être un peu présomptueux de notre part, c'est de faire le procès du passé en fait. C'est de prétendre pouvoir dire le droit sur des choses qui se sont produites il y a très longtemps. De ce point de vue-là, j'aurais une attitude beaucoup plus en retrait en disant effectivement qu'il faut essayer de trouver des agréments d'aujourd'hui sans prétendre trouver l'agrément qui est universellement recevable pour tous les temps et tous les espaces. Donc, encore une fois, peut-être réfléchir aux choses au cas par cas. En ce qui concerne la question d'Ataï, c'est une question intéressante et je crois que dans votre intervention, il faut faire la différence entre les restes humains et les objets culturels. Je crois que ce sont deux choses différentes.

5. Loi visant à autoriser la restitution par la France des têtes maories à la Nouvelle-Zélande et relative à la gestion des collections ( $n^{\circ} 2010-501$ du 18 mai 2010), parue au jo 114 du 19 mai 2010.

6. Il fait référence ici à une autre table ronde organisée au festival de Rochefort. 
D'ailleurs, on le voit bien dans cette histoire de tête également à Rouen. Il y a aussi cette proue [māori] et on voit bien qu'il y a une ligne qui sépare en fait ces deux catégories d'objets qui sont dans les musées et qui, pour moi, relèvent de deux types de réflexion, même s'il y a des ponts possibles.

En ce qui concerne Ataï, ce qui est très intéressant, c'est qu'on a là une identité individuelle. C'est-à-dire qu'on sait qui est cette personne, à la différence de ce que j'évoquais tout à l'heure sur l'anonymat des restes humains. Il avait été dit : quand on ne sait pas qui c'est, on ne peut rien faire! On ne peut pas agir sur l'individualité et donc revenir au clan, à l'espace précis dans lequel on doit le faire. D'une certaine manière, la coutume ne connait pas l'anonymat. On est toujours quelqu'un d'un lieu, d'un pied de cocotier, d'un pied de sapin, d'un théâtre, on n'est pas quelqu'un de partout! Sauf que nous vivons dans un monde qui, aujourd'hui, se globalise et qui nous perçoit comme Kanak, qu'on soit des îles, du nord, du centre ou du sud, on est tous Kanak. Nous-mêmes, nous avons tendance aussi à nous réfléchir, à nous penser de manière plus globale. Donc, les choses peuvent évoluer. La relation qu'on a de notre passé évolue aussi, me semble-t-il, et continuera d'évoluer au même rythme que ça évolue ici en métropole ou en Europe.

Ataï était tombé dans l'oubli, et dans l'enfer, peut-être, du muséum d'Histoire naturelle. C'est une tête qui a été coupée le $1^{\text {er }}$ septembre 1878 lors des opérations militaires en NouvelleCalédonie et la tête a été immédiatement mise dans du formol, qui est un alcool de préservation, envoyée à Nouméa puis en France où elle a été déposée. Ce sont les médecins de Marine, et c'est une époque où une partie des missions du médecin de Marine coloniale était de récolter des crânes. Pour les grandes comparaisons de l'époque, on commençait à faire des grandes collections de crânes, pour essayer de comparer l'humanité et en fait d'essayer de voir s'il y avait des permanences ou, au contraire, des différences, etc.

Donc c'est un processus qui est, de ce point de vue-là, à la fois un processus militaire puisqu'il s'agit de récupérer un crâne sur quelqu'un qui est tombé lors d'une opération et, en même temps, une visée aussi scientifique, puisqu'il s'agissait de voir effectivement s'il y avait des critères... si son analyse livrait des choses sur son cerveau.

Et c'est Broca, le professeur Paul Broca, un des fondateurs de la phrénologie, et de la médecine moderne aussi d'une certaine manière, qui a pratiqué l'autopsie et le crâne a été... enfin, la tête a été ouverte et elle a été analysée. Il y a eu une publication en 1879 à la Cité d'Anthropologie sur cette tête ${ }^{7}$.

Ensuite, le crâne a été montré lors de l'Exposition universelle de 1889 à Paris. Le crâne a été montré, non pas dans la section ethnologie, mais dans la section médecine. C'était, il rentrait avec d'autres restes humains et c'est-à-dire, à ce moment-là, en lisant le guide de cette exposition, on s'aperçoit qu'ils avaient aussi coupé les mains et qu'ils avaient présenté les mains d'Ataï avec une préparation permettant de montrer les tendons, etc.

Le crâne ensuite tombe dans l'oubli des collections, et dans les années 1980, au moment où le nationalisme kanak donne de sa voix chez nous, il y a des demandes qui sont faites auprès de l'administration pour savoir où est ce crâne et il y a des questions qui sont posées au Sénat d'ailleurs, des questions au gouvernement et le gouvernement répond à l'époque que le crâne est probablement perdu et qu'il a peut-être été détruit lors des bombardements de la Seconde Guerre mondiale et qu'on ne sait pas où il est et qu'il n'y a plus de crâne.

La question reste en l'état jusqu'en juillet 2011. Et probablement d'ailleurs, c'est une sorte d'onde de répercussion de l'affaire des crânes māori. Le Muséum national d'histoire naturelle retrouve $^{8}$ dans ses réserves ce crâne et le fait savoir d'ailleurs par ce jeune scientifique qui travaillait sur la question des crânes et la juridiction. Et le Muséum dit :

"Voilà, on a ce crâne et maintenant, on attend que les autorités calédoniennes viennent faire leur demande, etc. »

Donc, les choses ont changé en même temps de part et d'autre. Il faut voir aussi que depuis, entre la demande qui a été faite dans les années 1980 et les années 2000, il y a eu les accords de Matignon puis l'accord de Nouméa. Les relations ont changé entre la métropole et la Nouvelle-Calédonie. Et il est probable que cela ait quelque chose à voir avec l'amnésie soudaine puis le retour de mémoire du Muséum d'histoire naturelle.

Aujourd'hui, la question du crâne d'Ataï, me semble-t-il, est moins une question qui touche le Muséum d'histoire naturelle qui, en montrant et en disant ouvertement qu'il était à la disposition des ayants droits pour une restitution, finalement, c'est une question qui nous est renvoyée. Qui nous est renvoyée à nous en Calédonie, de savoir comment va-t-on gérer cette question. Car il faut inventer d'une certaine manière quelque chose qu'on n'a jamais fait pour l'instant, 
puisque quand quelqu'un décède, on l'enterre à la suite, dans la foulée de son décès, mais on ne gère pas des questions qui ont cent ans d'âge. Donc la grande question et les discussions qui ont lieu actuellement en Nouvelle-Calédonie, c'est des questions coutumières. C'est-à-dire qui va le faire? Qui est légitime pour parler au nom de ce vieux? Dans quel endroit allons-nous le mettre? Quelles sont les négociations à faire? Puisque c'est quelqu'un qui a été tué et décapité lors des opérations militaires, mais ça aurait été bien pratique qu'il ait été exécuté et la tête coupée par un soldat français. Malheureusement, c'est fait par un autre Kanak qui à l'époque travaillait et, disons, faisait partie des opérations de répression au côté de l'armée? ${ }^{9}$ C'est un supplétif exactement. Et donc, ça nous renvoie aussi à la question de notre propre responsabilité dans l'histoire et la manière dont cette histoire peutêtre ne peut pas être lue en noir et blanc avec les bons d'un côté, les méchants de l'autre.

Mais peut-être que ça nous interroge en fait sur le propre regard qu'on a sur notre passé et je trouve que cette question est une bonne manière d'interroger notre capacité à regarder le passé, à travailler également avec le Muséum d'histoire naturelle parce que ce sont des médecins qui gèrent encore ces collections. Ça explique aussi une manière, la différence qu'il y a entre ces collections qui ont été constituées au XIX ${ }^{\mathrm{e}}$ par des médecins et aujourd'hui, ces collections elles sont gérées par des gens qui ne sont pas médecins, comme moi. Et c'est vrai que j'ai un rapport aux restes humains qui n'est pas du tout de même nature que celui qui aurait été médecin et qui en a vu tout le temps et qui travaille avec, avec du vivant comme avec du défunt. Donc je pense que ça change aussi le type de rapport qu'on peut avoir avec ces collections.

En tout cas, c'est quelque chose d'assez passionnant. Je sais que les discussions sont en cours dans la région de La Foa, mais que c'est aussi en cours dans la région de Canala et que ça met aussi en mouvement tous les supplétifs [leurs descendants] qui étaient... Alors, tous les insurgés et tous les supplétifs de l'époque. Donc c'est une question vraiment fondamentale pour nous, je pense, et c'est intéressant là encore que ça soit depuis un musée, finalement, que le questionnement nous est renvoyé sur notre capacité aujourd'hui à regarder en face notre passé et à trouver des solutions qui ne peuvent pas être des solutions du passé, qui ne sont que des innovations, des manières modernes de traiter. Et peutêtre que ce qui a été initié en Nouvelle-Zélande, avec cet espace commun pour les morts anonymes, pour les crânes qui sont revenus, c'est peut-être une idée aussi et sur laquelle on peut travailler nous en Calédonie pour traiter cette question du crâne d'Ataï.

Sébastien Minchin. Je voulais juste dire que c'est exactement dans cet esprit-là qu'on avait abordé la démarche. C'est-à-dire que l'objectif, ce n'était pas dès le départ d'amener une quelconque culpabilité sur ce qui s'était fait par le passé. Il n'y a pas de blanc ou de noir, il n'y a pas de bon et de méchant. Ce n'est pas ça. On sait que, comme le disait Georges, on était dans un conflit, dans des guerres, bon, quil y a forcément des gens dans tous les camps. Chacun fait comme il peut par rapport à ses armes, par rapport à ses moyens. Et, dès le départ, nous, ce n'était pas du tout de dire "Il y a eu ce trafic". C'est vrai qu'il y a eu ce trafic. Mais peut-être qu'à l'époque, $j$ 'aurais vendu père et mère pour avoir une tête māori. Voilà.

Aujourd'hui, les choses ont évolué, on peut parler de cette histoire. Le fait de les restituer et de les remettre dans le cœur du débat, cela a permis aussi de faire avancer la science et la recherche par rapport à ces questions. En gros, pratiquement toutes les têtes qui sont arrivées au XIX ${ }^{e}$ siècle, en France, ont toutes été remisées, soit en exposition, soit dans les réserves des musées jusqu’en 2007. Ce n'est qu’à partir de 2007 qu'on aborde la démarche où les têtes reprennent du service et où on va commencer à les étudier, à travailler dessus, à les modéliser, à travailler avec les tatoueurs māori et à faire que, d'un seul coup, tout le monde s'en empare et participe. Et comme vous le disiez, Ataï, c'est exactement le même principe. C'est-à-dire qu'à un moment, on se rend compte qu'on a quand même pas mal de restes humains et, que du coup, il faut aussi qu'on sache ce que l'on a comme restes humains. Et Ataï ressort des placards.

Et je me souviens de ce moment-là parce que le jour de la cérémonie de restitution, en 2011, était présent quelqu'un que vous connaissez peut-être, qui est un auteur qui s'appelle Didier Daeninckx, qui avait écrit Cannibales et Le retour d'Ataï et qui nous a suivis en fait tout au long de cette démarche pendant toutes ces années. Et il était présent le jour de la cérémonie de restitution à Rouen. Et il m’a dit :

"Sébastien, je viens de vivre deux moments fabuleux en deux jours. Là, aujourd'hui, la restitution de la tête pour laquelle on a essayé de se battre et puis on a avancé dans un dialogue avec les uns et les autres et puis hier, on m'a montré la tête d'Ataï. "

Et du coup, voilà, c'est ça qui est aussi intéressant et c'est ça qu'on voulait mettre en avant. C'est de dire : 
" À un moment, servons-nous de ces éléments-là pour avancer ensemble, pour dialoguer ensemble sur ces problématiques-là et en montrant que ce n'est pas si simple que ça ».

Claude Stéfani. Je voudrais quand même dire que pour nous, conservateurs, le débat dépasse aujourd'hui la très difficile problématique des restes humains. Tout à l'heure, on évoquait la pirogue. En fait, je crois que tous ces objets qui viennent de ces cultures doivent être reconsidérés. Il faut changer notre regard et notre attitude, notre posture. Et Denis [Roland] a parlé justement du respect. Je crois que c'est vraiment cela : il faut les considérer autrement que comme de simples pièces qui auraient seulement une valeur esthétique. D'où l'intérêt, en effet, quand on peut le faire, de demander aux descendants de ceux qui les ont fabriqués, quelle est leur signification, leur valeur, afin que nous puissions les traiter différemment de ce qu'ont pu faire nos devanciers par rapport justement à la gestion des têtes māori. Là, les avis diffèrent. On a bien vu qu'il y a des attitudes radicalement différentes dans le mode d'appréhension, de présentation et de conservation de ces objets dans les musées. Je crois que c'est un débat qui dépasse le cadre des restes humains. Et c'est pour cela que j'évoquais la crainte des conservateurs de voir des réclamations qui porteraient sur autre chose que des restes humains. Mais je crois qu'il faut travailler nous-mêmes, en tant que professionnels des musées, sur ce type de gestion par des échanges avec les populations autochtones. Cela a été le cas à Rouen, cela a été le cas à Rochefort, je le rappelle, avec les Kanak. Et je pense qu'il faut aller dans ce sens-là pour qu'il y ait plus de réflexion par rapport aux appropriations qu'on s'est autorisées pendant très très longtemps. Aujourd'hui, il y a une façon de rendre les choses, peut-être pas par la rétrocession matérielle, mais en tout cas par nous, par un travail différent afin que ces objets retrouvent une plus grande signification et que ce soient plus que des simples objets de musée morts et qu'on leur redonne une seconde, ou une valeur différente. Pardon?

(Personne dans la salle : Une valeur commune.)

Sébastien Minchin. Une valeur commune, absolument. Une valeur commune et, ce qui m'agace beaucoup - je terminerai là-dessus -, c'est un mouvement complètement inverse auquel on assiste en ce moment, enfin déjà depuis quelque temps, où on voit dans le commerce de l'art dit primitif ou premier, des objets qui sont traités comme de purs objets d'art et qui sont complètement dépourvus, souvent, de toute valeur ancestrale. Enfin, on le voit quand il y a des foires d'art primitif, on voit, n'est-ce pas, alors des restes humains qui sont vendus ou des objets extrêmement sacralisés par les populations qui les ont produits et qui sont vendus pour leur seule valeur esthétique.

Il n'y a pas très longtemps, il y a eu une vente à Paris de masques hopi qui, en amont, avait été contestée justement par la population amérindienne en question car c'était un patrimoine très important $t^{10}$. Et ils ont été déboutés bien sûr mais il y a une association qui a réussi à en racheter deux pour les rétrocéder. Mais je crois, qu'en tout cas, c'est peut-être maintenant justement le rôle des musées de dire "ça suffit "! Il faut que nous, on joue ce rôle je dirais d'interlocuteur privilégié avec les populations.

Gilles Luneau. En fait, c'est vraiment le rapport à la mort ou aux traces de la mort dans les musées qui redéfinit aujourd'hui la fonction même du musée. Mais peut-être que dans cette civilisation dont Georges Nuku disait qu'elle globalise la médiocrité, c'est au musée qu'on va se ressourcer et retrouver des valeurs pour se régénérer finalement. Donc on n'est pas loin du cycle de vie et de mort.

Sébastien Minchin. L’avenir passera par les musées.

Intervenant. C'est la question du retour des restes humains on va dire concernant les têtes, là. Mais de l'autre côté du droit, on insiste à ce que le patrimoine français, il soit gardé dans les musées aussi, c'est réglementé. Quel est le considérant, enfin au niveau de chacun d'entre vous spécialistes, si vous avez eu le cas vraiment? Bon à part les restes humains. Nous, en Calédonie par exemple, Emmanuel s'en rappelle ou il se souvient bien, les autorités douanières néocalédoniennes ont surpris ce voilier qui venait du Vanuatu avec en double fond des sculptures, des œuvres ni-vanuatu et elles sont encore chez nous, je crois. On leur a demandé. Bon, il n'y a pas eu cette identification auprès de familles de clans au Vanuatu et il y a deux débats là-bas qu'on garde. Parce que c'est du patrimoine français et ça a été surpris dans nos eaux, ça a été récolté dans nos eaux par la douane et de l'autre côté, là-bas, enfin même nous, l'autre considérant c'est qu'il faut rendre ces choses, ces pièces au Vanuatu. Et ce ne sont pas des restes humains, mais voilà.

De votre côté, entre le patrimoine qui est, enfin, considéré et puis ça... Bon, Ataï, c'est spécifique parce qu'on a identifié les familles. Voilà. Côté français et maintenant du côté d'Élisabeth,

10. Voir, par exemple, http://blogs.mediapart.fr/blog/babou78/030513/des-masques-katsinam-drouot-retour-sur-uneenchere-marquee-par-beaucoup-dincomprehension-dignorance-et. 
comme disait Georges, est-ce que le cas est avéré chez vous où des familles et des clans, des nations māori ont repéré, ont identifié les têtes ? Et comment ça se passe du côté anglais, pour qu'on rapatrie ou pas?

George Nuku. Il y a des spéculations qui sont en train de se faire et, à mon avis, ce sont des questions qui sont dangereuses. Il y a trop de facteurs, c'est trop complexe. Et donc c'est dangereux d'essayer de simplifier à mon avis. Je voulais aussi m'exprimer sur la question des ancêtres, je vous soumets mon avis que ces têtes n'étaient pas prêtes à rentrer chez elles. Elles ont eu la force d'être dans une boîte pendant des centaines d'années et elles pouvaient rester là pendant plusieurs centaines d'années. Elles représentent un contexte et une histoire et c'est ça qui est important pour moi. J'ai essayé de communiquer l'idée qu'elles représentent la capacité des Mãori de faire face à la mort, de faire face à la peur de mourir. Et cette confrontation est une manière de vivre, de considérer chaque jour comme si c'était le premier et le dernier jour. Elles nous donnent cette capacité. Et maintenant qu'elles sont retournées chez elles, ce n'est plus possible. D'une certaine manière, on a perdu quelquesunes des meilleures cartes qu'on avait dans la main, on les a abandonnées. Elles représentent la possibilité pour les publics de se regarder euxmêmes, de méditer sur leurs propres vies, et de se poser des questions. Cette possibilité-là on l'a perdue.

Moi, je vais continuer le "trafic des têtes", je vais les faire en plexiglas, mais elles représentent les mêmes idées et concepts que les vraies têtes. Et les gens de chez moi les ont ramenées avec les cérémonies qu'il fallait en Nouvelle-Zélande et je vais m’assurer que le débat continue avec des cérémonies aussi. Tant que j'aurais mes neuf doigts et demi, je continuerai à faire cela. Et je suis prêt à donner ma vie pour cela. Cela m'a déjà coûté une partie de mon corps et cela m'a coûté beaucoup personnellement ; pour quelqu'un comme moi, c'est mon chemin, c'est mon destin. Et quand on est choisi, on n'a pas le choix. Le choix a déjà peut-être été fait pour moi.

Pour moi, la question la plus importante, c'est de parler de cela aux jeunes, d'essayer d'aller audelà du macabre et de voir la beauté et la force ; de voir tous ces paradoxes que j'ai essayé de mettre en mots. C'est là que la vérité se fait, c'est là que la vérité est entre la vie et la mort, entre le mal et le bon. La vérité est une découverte, ce n'est pas quelque chose d'absolu. Et pour moi, c'est une occasion ratée. Donc je vais continuer à essayer de remplacer ces espaces vacants avec de nouveaux ancêtres. Et d'une certaine manière, sans vraiment le vouloir, les Māori sont tombés dans ce piège de traiter les ancêtres à la manière des Européens, de les voir comme quelque chose

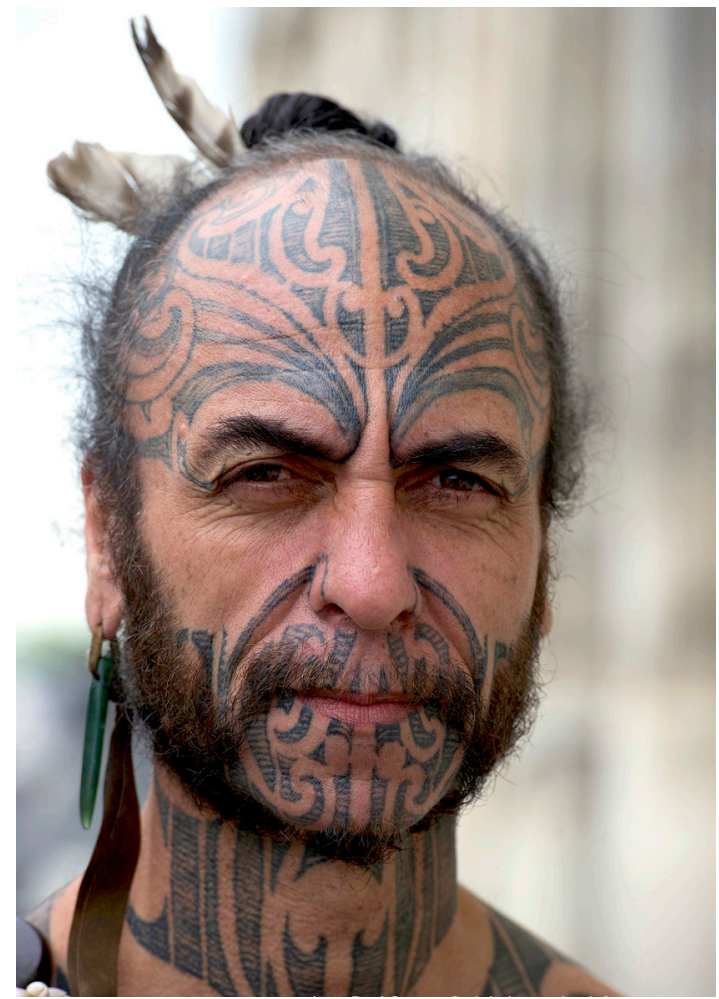

Рното 4. - George Nuku (cliché de Paol Gorneg () globalmagazine.info)

dont on a peur. Le fait qu'ils ne voulaient pas voir cette tête à Rouen, j'en comprends les raisons, mais moi, j'étais, je suis préparé à prendre tous les risques imaginables pour voir cette tête. Parce que ma tête aussi est tatouée. Cela pose des questions aussi sur ma propre tête. Qu'estce qu'il va se passer quand moi je m'en irais? Qu'est-ce ma tête va s'organiser comme exposition? Est-ce que ceux qui sont à côté de moi vont faire du bon travail avec ma tête ? Combien est-ce que mes descendants vont acheter d'armes avec ma tête?

Je sais que je donne l'impression de traiter cela avec désinvolture, mais tout cela est très sérieux. C'est ça la question des toi moko en NouvelleZélande. Aucun des hommes dont la tête est tatouée complètement n'est pour l'instant décédé. Si nous voulons pouvoir revendiquer la coutume, la pratique de tatouer les têtes, comme l'est ma tête, il faut se poser la question de "Comment est-ce qu'on meurt? Comment est-ce qu'on détermine les coutumes qui accompagnent la mort ? ». La loi actuelle permet seulement d'être enterré dans un endroit particulier ou sinon, être inhumé. Peut-être que c'est la même chose en France. Toutes ces questions se posent, voilà. Quelles sont les coutumes qu'il va falloir réhabiliter et quelles sont les coutumes qui doivent rester enterrées ? Parce que si on arrive à déterminer comment on meurt, ça nous aide aussi à déterminer comment on vit. 
Intervenante. Je vais parler en français. Merci d'avoir partagé votre vision, votre opinion sur les têtes et votre manière de leur rendre hommage en fait à travers l'agentivité que vous voyez dans ces têtes, ce qui est une autre façon de rendre hommage, je pense, aux ancêtres. Ma question, c'est la première fois que j'entends une perspective comme la vôtre, et je me demandais à quel point est-ce qu'elle est partagée en Nouvelle-Zélande et s'il y a eu un débat public au sein de la communauté māori pour savoir quel était le sort réservé à ces têtes?

George Nuku. Il y a différents avis et des contradictions. La communauté māori n'a pas un seul point de vue sur cette question. Il y a des anciens et des communautés qui sont en colère et ils pensent que le musée a pris les décisions tout seul sans consulter les tribus. Et au sein des tribus, ils disent qu'il y a des représentants du musée qui ne parlent pas forcément pour eux. Mais on adore ne pas être d'accord. Mais je respecte et je soutiens les raisons pour lesquelles le musée Te Papa a cette position et je respecte aussi les raisons pour lesquelles il y a eu une restitution. Et je soutiens ce point de vue, mais je dis juste que ce n'est pas la seule perspective sur la question.

Quand les têtes sont rentrées à Wellington, à Te Papa, elles ont été accueillies par des larmes, par la parole, mais il n'y a pas eu d'échanges. Qu'est-ce qu'on rend, nous, aux musées? Qu' est-ce qu'on donne en échange de ce qu'il nous a été donné ? Un simple merci n'était pas suffisant. On était censés donner quelque chose jusqu'à ce que ça nous fasse mal parce que c'est ça qui détermine la valeur de ce que nous recevons. Et ça donne aussi aux artistes du travail, des artistes comme moi, pour remplacer les vieux trésors par de nouveaux trésors. Quelles sont les attentes qu'auront nos descendants dans cent ans? Qu'est-ce qu'ils feront? Et ça, ce serait un très bel échange qui se ferait pour l'éternité et qui se referait et qui se referait. Cet échange réciproque, c'est ça qui fait que la roue continue de tourner. Donc j'espère qu'il y aura des volontés de donner au musée de nouveaux trésors.

La question a été soulevée dans le film de " $\mathrm{La}$ proue de la pirogue ${ }^{11}$. Ça, ça a plus de valeur que l'ancêtre parce qu'il avait plus de choses impliquées dans la création. Donc, faites attention, quand vous adoptez ce positionnement moral occidental par rapport aux restes humains, faites attention! Excusez-moi de dire cela, mais pour moi tout ça c'est un peu du Disneyland. Il y a trop de bons sentiments, c'est bien charmant tout ça, mais je me dis que pour certaines personnes qui étaient impliquées dans la restitution, que ce soit ici ou que ce soit là-bas, c'était un jeu politique. Et moi aussi, d'une certaine manière, je fais ce que je fais à des fins politiques. Puisque je veux être connu, je veux amener du prestige à mon marae, à ma communauté et le pire qui puisse arriver à un Māori, c'est d'être oublié. Donc je vais continuer à faire des choses à faire des têtes, à faire des pirogues et je vais m'assurer qu'elles soient dans tous les musées du monde si c'est possible. À moins que je n'en vienne à n'avoir plus de doigts...

Donc comme vous l'avez dit, la question qui est importante, c'est la question de l'agentivité, $\mathrm{du}$ fait que les hommes peuvent être acteurs de leur destin. Il faut que le passé soit devant nos yeux, pour qu'on ne le répète pas et ça, c'est quelque chose qui est valable pour tout le monde. Il faut qu'on s'approprie notre passé. En regardant notre passé en face, ici en France, cela nous permet d'avoir des dialogues ici que peutêtre on ne peut pas avoir chez nous. Cela permet aux communautés issues du peuplement colonial, aux communautés autochtones, de ne pas être dans une impasse de dialogue, une impasse dans laquelle on ne peut plus parler des choses quand on est chez nous.

Dans les années 1980, il y a une exposition très connue qui est allée aux États-Unis ${ }^{12}$ et cela a changé la manière dont les gens réfléchissaient en Nouvelle-Zélande. Parce que le monde entier a vu l'art et le succès des productions culturelles māori qui étaient au même niveau que les productions internationales. Jusqu'à ce momentlà, ce n'était pas reconnu en Nouvelle-Zélande. Donc il fallait qu'on aille d'abord à l'extérieur de la Nouvelle-Zélande pour ensuite avoir une reconnaissance en Nouvelle-Zélande. Et c'est quelque chose que je dois faire moi aussi. N'oubliez pas que Te Papa est une institution. Moi aussi, George Nuku, je suis une institution. L'institution de moi-même.

Gilles Luneau. Merci Georges. La dernière question? Une, deux ? Deux questions.

Intervenante. Je voudrais juste apporter un petit témoignage de ce que peuvent faire les musées pour prêter des ouvres. Je pense qu'ici, des intervenants pourront en parler, notamment Véronique Mu qui était (ancien conservateur du musée) à Tahiti. C'est le retour du Panthéon mangarévien en Polynésie, à Tahiti, au musée de Tahiti et des îles. Il y avait notamment un dieu, Rongo, qui était au musée de La Rochelle. C'était un moment très émouvant. Le Panthéon mangarévien, c'est donc les statuettes qui représentaient les dieux polynésiens qui avaient été pour la plupart détruites par les pères de Picpus, mais ils en avaient sauvé quelques-unes qu'ils avaient

11. Toujours conservée au musée de Rouen.

12. Voir l'exposition Te Tapa qui a circulé aux USA en 1985-1986 (Saint-Louis, Californie, Chicago). 
envoyées en Europe. Aujourd'hui, elles sont disséminées au British Museum de Londres, à Rome, un petit peu partout, il y en a en France également au musée du quai Branly et elles sont revenues, une dizaine d'entre elles sont revenues à Tahiti pour une exposition. Les gens, certains, une délégation de Mangareva sont venus pour les voir et voilà, c'était un moment très émouvant. Je pense que certains intervenants pourraient évoquer cette chose-là qui devrait se faire, on parlait de prêts à la place des restitutions. Cela fait partie de ces choses qui sont très difficiles à monter et qui, pourtant, ont un grand impact.

Gilles Luneau. Merci pour le témoignage. Une dernière question, Monsieur?

Intervenante. Merci de me donner la parole. Je ne pouvais pas rester assis sans partager un petit bout de parole pour faire raisonner la voix de la Mélanésie aussi avec nos cousins et frères polynésiens. Donc je vais dire un petit mot dans la langue [...] de la région d'où aussi est issue le grand chef Ataï dont on a parlé ce soir ou aujourd'hui en tout cas. Et pour dire qu'à travers cette langue, Ataï vit toujours en nous. [...] Voilà, je disais qu'il y a un seul feu du soleil qui nous tire et c'est ce même feu de soleil qui nous a guidés. Que nous vivons, qu'il n'y a qu'une seule vie et un seul souffle. On sort d'un même lieu, on appartient au même lieu, à un seul lieu. Et la parole, c'est la parole des constellations d'étoiles qui nous ont toujours veillés et qui font toujours qu'on existe aujourd'hui. Voilà.

J'avais envie aussi de faire une proposition aux hommes des musées qui sont ici, de la même sorte qu'une proposition aux hommes de pensée, aux hommes d'intelligence qui sont aussi présents dans cette salle. Monsieur disait que peutêtre, le musée serait le futur de nos sociétés. On cherche un endroit où mettre les dépouilles, les ossements à partir de tout ce qu'on pose comme questions aujourd'hui.

Une proposition, je ne sais pas si c'est une bonne ou une mauvaise, le futur musée qui éduquerait nos futures générations, j'y crois, j'y crois, je suis moi-même aussi un artiste aussi et $j$ 'espère que ce futur musée existera un jour et pourquoi pas, on parlait d'Ataï. Si Ataï doit revenir chez nous, dans mon pays [...].

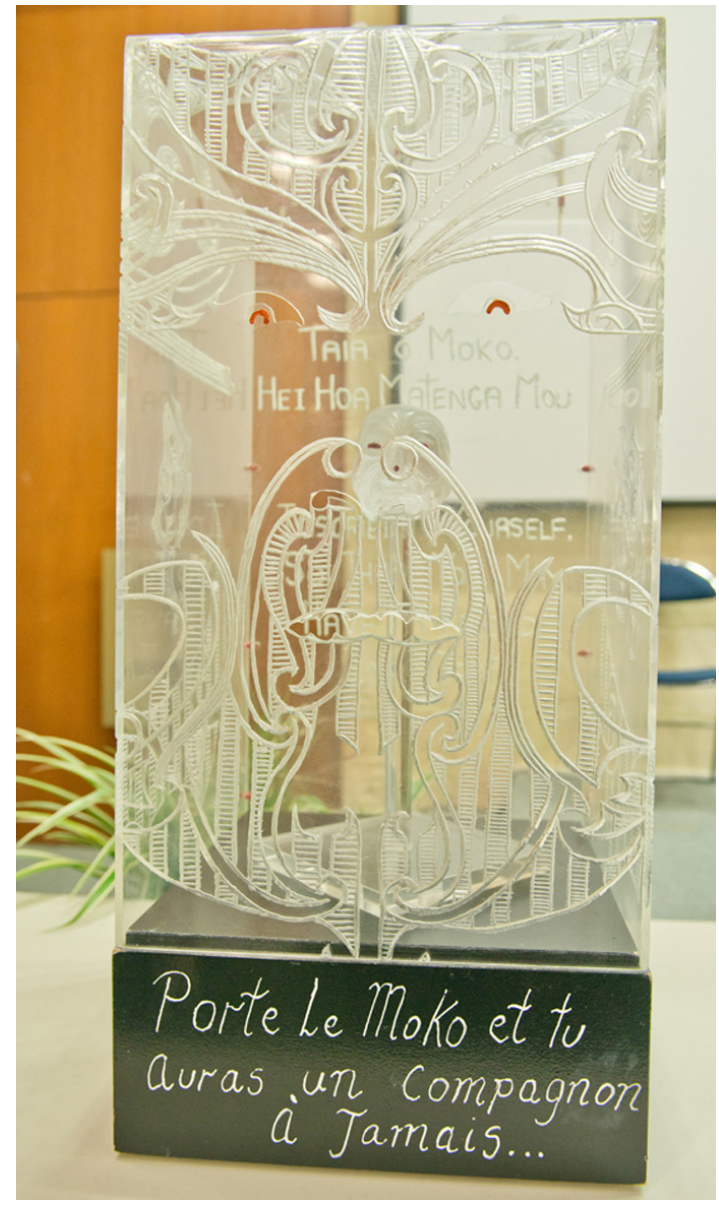

Рното 5. - Sculpture de Georges Nuku (cliché de Béatrice Téraube)

Tout à l'heure, Monsieur Kasarhérou disait que c'est très difficile parce que ça réveille beaucoup de vieilles histoires, mais comme disaient les grands frères aborigènes hier dans l'autre film, ces histoires-là nous regardent. Ce sont nos histoires à nous. Et s'il faut faire venir le vieux Ataï chez lui, pourquoi pas trouver un endroit. Et chez nous, on a des églises qui ont été posées par la colonisation. Pourquoi ces églises ne deviendraient pas un jour de futurs musées modernes pour toutes les générations ? Voilà, merci.

Gilles Luneau. Merci bien. Bon. Je vous propose de remercier maintenant pour conclure tous nos invités, tous particulièrement Georges venu de si loin, il est bienvenu, lui et tous les Māori, à Rochefort, on renouvelle l'invitation. 